levels of C4M, C6M, and CRPM at AE-IIP were positively correlated with \%FVC $(r=0.37, p=0.008 ; r=0.36, p=0.011 ; r=0.33, p=0.020$; respectively). Serum levels of C4M $(p=0.002)$ and C6M $(p=0.024)$ were increased while ELM7 $(p=0.024)$ and VCANM $(p<0.0001)$ were decreased at AE-IIP as compared with $S$-IIP when analyzing all patients. IPF patients had lower levels of VCANM at AE-IPF $(p=0.0001)$. Patients with paired samples showed significantly elevated serum levels of C4M ( $p=0.004)$ and decreased levels of ELM7 and VCANM ( $p=0.036$ and $p=0.0001$, respectively) at AE-IIP. C1M and C6M levels at AE-IIP were borderline related to mortality outcome for IPF patients (both $p=0.059$ ) with levels above median associated with a higher risk of mortality. Analyses of all or paired patients showed no associations with mortality.

Conclusions: Serological levels of neo-epitope biomarkers of ECM degradation were associated with AE-IIP and weakly with mortality outcome. These results indicate that the rate of ECM remodeling in the lungs of patients with IIP is significantly altered during periods of high disease activity such as an acute exacerbation. The difference in degradation profile for the proteins studied is intriguing and indicate activation of different processes contributing to AE-IIP. Neo-epitope biomarkers of the ECM might be useful in identifying patients diagnosed with rheumatic disease as SSc and AS with pulmonary involvement. Acknowledgements: The Danish Research Foundation

Disclosure of Interest: None declared

DOI: 10.1136/annrheumdis-2017-eular.5699

\section{AB0181 DIFFUSING CAPACITY AND CLINICAL CHARACTERISTICS OF PATIENTS WITH SYSTEMIC SCLEROSIS - DATA FROM THE GERMAN NETWORK FOR SYSTEMIC SCLEROSIS}

P. Moinzadeh ${ }^{1}$, N. Blank ${ }^{2}$, E. Siegert ${ }^{3}$, J. Henes ${ }^{4}$, M. Worm ${ }^{5}$,

C. Sunderkoetter ${ }^{6}$, M. Schmalzing ${ }^{7}$, A. Kreuter ${ }^{8}$, C. Gunther $^{9}$, L. Susok ${ }^{10}$, G. Zeidler ${ }^{11}$, I. Koetter ${ }^{12}$, U. Mueller-Ladner ${ }^{13}$, T. Krieg $^{1}$, A. Juche ${ }^{14}$,

T. Schmeiser ${ }^{15}$, G. Riemekasten ${ }^{16}$, E. Aberer ${ }^{17}$, N. Gaebelein-Wissing ${ }^{18}$, J.H.W. Distler ${ }^{19}$, M. Sárdy ${ }^{20}$, C. Pfeiffer ${ }^{21}$, K. Kuhr ${ }^{22}$, N. Hunzelmann ${ }^{1}$,

F. Bonella ${ }^{23}$, M. Kreuter ${ }^{24}$. $^{1}$ Dermatology, University Hospital Cologne, Cologne;

${ }^{2}$ Rheumatology, University Hospital Heidelberg, Heidelberg; ${ }^{3}$ Rheumatology, Charité Universitätsmedizin Berlin, Berlin; ${ }^{4}$ Rheumatology, University Hospital Tuebingen, Tuebingen; ${ }^{5}$ Dermatology, Charité Universitätsmedizin Berlin, Berlin ${ }^{6}$ Dermatology, University Hospital Muenster, Muenster; ${ }^{7}$ Rheumatology, University Hospital Wuerzburg, Wuerzburg; ${ }^{8}$ Dermatology, HELIOS St. Elisabeth Klinik Oberhausen, Oberhausen; ${ }^{9}$ Dermatology, University Hospital Carl Gustav Carus, Dresden; ${ }^{10}$ Dermatology, St. Josef Hospital Bochum, Bochum; ${ }^{11}$ Rheumatology, Johanniter-Krankenhaus im Fläming Treuenbrietzen, Treuenbrietzen; ${ }^{12}$ Rheumatology, Asklepios Klinik Altona, Hamburg:

${ }^{13}$ Rheumatology, Justus Liebig University Giessen, Kerckhoff Clinic, Bad Nauheim; ${ }^{14}$ Immanuel Krankenhaus Berlin-Buch, Berlin; ${ }^{15}$ Rheumatology, Krankenhaus St. Josef, Wuppertal: ${ }^{16}$ Rheumatology, University Medical Center-UKSH, Luebeck, Germany; ${ }^{17}$ Dermatology, Medical University of Graz, Graz, Austria; ${ }^{18}$ Dermatology, HELIOS University Hospital Wuppertal, Wuppertal; ${ }^{19}$ Rheumatology, University Hospital Erlangen, Erlangen; ${ }^{20}$ Dermatology, Ludwig Maximilians University Hospital, Munich; ${ }^{21}$ Dermatology, University Medical Center UIm, UIm; ${ }^{22}$ IMSIE, University of Cologne, Cologne; ${ }^{23}$ Unit for interstitial lung diseases, Ruhrlandklinik University Hospital Essen, Essen; ${ }^{24}$ Center for interstitial and rare lung diseases, Thoraxklinik, University Hospital Heidelberg, Heidelberg, Germany

Background: Lung involvement, i.e. interstitial lung disease (ILD) and pulmonary hypertension $(\mathrm{PH})$, is common in patients with systemic sclerosis ( $\mathrm{SSc}$ ), significantly limiting quality of life and survival. Data on clinical correlations between lung function and clinical subsets of SSc are sparse.

Objectives: To investigate the relationship of DLCO and clinical characteristics in patients SSc patients within the registry of the German Network for Systemic Scleroderma.

Methods: Clinical data of the patient registry, currently including DLCO data of 1917 patients were evaluated. In total, these patients were clinically evaluated 5997 times (i.e., at the first visit and during follow-up visits). At the initial visit and during follow-up DLCO levels were correlated with clinical characteristics.

Results: At initial presentation, $64 \%$ of the patients had DLCO levels $<75 \%$ predicted. Impaired DLCO levels were observed in $74 \%$ of dcSSc patients, in $64 \%$ of SSc-Overlap patients and $57 \%$ of IcSSc patients $(p<0.0001)$. Furthermore, male patients $(62 \%)$, patients with $\mathrm{PH}(80 \%)$, ILD $(80 \%)$, dyspnea $(78 \%)$, and those with presence of anti-topoisomerase I antibodies $(71 \%)$ exhibited significantly more often DLCO levels $<75 \%(p<0.01)$. Patients suffering from dcSSc had the lowest DLCO levels (mean value, $62 \%$ ), followed by patients with SSc-Overlap syndromes (mean value, 67\%) and IcSSc patients (mean value, $70 \%$ ) revealing significant differences between subsets. Long-term follow-up evaluation (mean follow up, 6.0 years) revealed that in comparison to IcSSc patients dcSSc patients (OR 2.1; $p<0.0001 ; 95 \%>C l$ 1.7-2.5) and SSc-Overlap patients (OR, 1.55; $\mathrm{p}<0.0001 ; 95 \% \mathrm{Cl}, 1.2-2.0)$ had a significantly increased risk to a decrease in DLCO levels $<75 \%$.

Conclusions: Impairment of pulmonary function as determined by diffusing capacity DLCO is more common and more pronounced in patients with dcSSc and SSc-Overlap Syndrome compared to IcSSc. DLCO may be useful for diagnosing and monitoring pulmonary involvement in SSc.

Disclosure of Interest: None declared
DOI: 10.1136/annrheumdis-2017-eular.3474

\section{AB0182 IN VITRO CHARACTERIZATION OF DERMAL FIBROBLASTS FROM SYSTEMIC SCLEROSIS PATIENTS}

R. Brizzolara $^{1}$, P. Montagna ${ }^{1}$, S. Soldano ${ }^{1}$, A.C. Trombetta ${ }^{1}$, B. Ruaro ${ }^{1}$, A. Sulli ${ }^{1}$, S. Scabini ${ }^{2}$, E. Stratta ${ }^{2}$, V. Smith ${ }^{3}$, M. Cutolo ${ }^{4} .{ }^{1}$ Research Laboratory and Academic Division of Clinical Rheumatology, Department of Internal Medicine, University of Genoa, IRCCS San Martino; ${ }^{2}$ Oncologic Surgery, Department of Surgery, IRCCS San Martino IST, Genoa, Italy; ${ }^{3}$ Department of Rheumatology, Ghent University Hospital, Ghent, Belgium; ${ }^{4}$ Research Laboratory and Academic Division of Clinical Rheumatology, Department of Internal Medicine, University of Genoa, Genoa, Italy

Background: Systemic sclerosis (SSc) is characterized by progressive fibrosis of the skin and/or internal organs. Skin fibrosis is mainly due to the excessive production of extracellular matrix (ECM) proteins from dermal activated fibroblasts. The high amounts of transforming growth factor-beta (TGFbeta) production induce fibroblast proliferation and their transition into profibrotic myofibroblasts. Consequently, the increased presence of alpha-smooth muscle actin (alphaSMA)-positive myofibroblasts in affected tissues is associated with fibrotic tissue remodelling, that characterize SSc [1-4].

Objectives: To characterize dermal fibroblasts from SSc patients by evaluating the gene expression of specific phenotypic and profibrotic markers: fibrillar collagen type I (COL I), fibronectin (FN), fibroblast-specific protein 1 (S100A4), TGFbeta and alpha-SMA (marker of myofibroblasts).

Methods: Human dermal fibroblasts were obtained by skin biopsy from affected areas of 6 active IcSSc patients after written informed consent and approval of medical ethics committee. SSc patients fulfilled the new EULAR/ACR criteria for SSc and they were treated only with various vasodilators (no severe clinical SSc complications were present at the time of skin sampling). Human SSc dermal fibroblasts were cultured up to $80 \%$ of confluence and at the third to fifth subpassages were used for experiments. Total RNA was extracted and quantitative real-time PCR (qRT-PCR) was performed to evaluate the gene expression of relevant fibroblast markers: COL I, FN, S100A4, TGFbeta and alpha-SMA.

Results: In human SSc fibroblasts the gene expression of the relevant markers $\mathrm{COL} \mathrm{I,} \mathrm{FN} \mathrm{and} \mathrm{S100A4} \mathrm{resulted} \mathrm{of} 10^{7}$ RNA copies for each gene. Interestingly, in the same tested fibroblasts, the profibrotic alpha-SMA gene expression (marker of myofibroblasts) was similarly found highly expressed, with a result of $10^{7}$ gene copies. On the contrary, TGFbeta showed a much lower gene expression $\left(10^{3}\right.$ copies) compared to the other investigated genes.

Conclusions: In human SSc cultured dermal fibroblasts, the gene expression of ECM proteins ( $\mathrm{COL} I$ and $\mathrm{FN}$ ), was found associated with a relevant gene expression of S100A4 and alpha-SMA, confirming their myofibroblast phenotype, as highly differentiated. Interestingly, TGFbeta gene appears to be less expressed. It could be concluded that myofibroblasts from the SSc skin mainly undergo the effects of the tissutal TGFbeta, since it seems that they do not contribute to its further production once differentiated.

References:

[1] Cutolo M. et al. J Rheumatol 2015;42:3.

[2] Brunasso A.M. et al. F1000Research 2016;5:723.

[3] Hinz B et al. Am J Pathol 2012;180:1340-55.

[4] Dumoitier N. et al. Arthritis Rheumatol 2016 [Epub ahead of print].

Disclosure of Interest: None declared

DOI: 10.1136/annrheumdis-2017-eular.3687

\section{AB0183 ISOLATION AND CHARACTERIZATION OF CIRCULATING FIBROCYTES FROM SYSTEMIC SCLEROSIS PATIENTS: AN VITRO INVESTIGATION}

R. Brizzolara ${ }^{1}$, P. Montagna ${ }^{1}$, S. Soldano ${ }^{1}$, A.C. Trombetta $^{1}$, P. Contini ${ }^{2}$ V. Smith ${ }^{3}$, B. Ruaro ${ }^{1}$, A. Sulli ${ }^{1}$, M. Cutolo ${ }^{1}$. ${ }^{1}$ Research Laboratory and Academic Division of Clinical Rheumatology, Department of Internal Medicine, University of Genoa, IRCCS San Martino; ${ }^{2}$ Division of Clinical Immunology, Department of Internal Medicine, University of Genoa, Genoa, Italy; ${ }^{3}$ Department of Rheumatology, Ghent University Hospital, Ghent University, Ghent, Belgium

Background: In physiological and pathological conditions, such as in tissue repair and in fibrosing diseases (i.e. systemic sclerosis, SSc), the importance of circulating fibrocytes relies on the capacity of such cells to migrate into scleroderma-affected tissues (through CXCR4/CXCL12 interaction) and to differentiate into fibroblasts/myofibroblasts, inducing fibrosis (through matrix protein deposition) (or both) [1-3]. In addition, fibrocytes seem to exert immunomodulatory effects, expressing class II major histocompatability complex molecules (HLA-DP, -DQ, and -DR) [4].

Objectives: To isolate fibrocytes from Circulating Progenitor Cells (CPCs peripheral blood mononuclear cells PBMCs) of SSc patients and healthy subjects and to identify them by fluorescence-activated cell sorter analysis (FACS), using their specific markers: the leukocyte common antigen CD45, collagen I (COL I), the chemokine receptor CXCR4 and HLA-DR [2]

Methods: Samples were collected, at basal time (t0), from 11 SSc patients, affected by SSc (treated only with different vasodilator drugs) and 5 healthy 